\title{
BOUNDS ON THE STANLEY DEPTH AND STANLEY REGULARITY OF EDGE IDEALS OF CLUTTERS
}

\author{
YI-HUANG SHEN
}

\begin{abstract}
Let $I$ be the edge ideal of a clutter $\mathcal{C}$ in a polynomial ring $S$. In this paper, we present estimations of the Stanley depth of $I$ as well as the Stanley regularity of $S / I$, in terms of combinatorial data from the clutter $\mathcal{C}$.
\end{abstract}

1. Introduction. Depth, projective dimension and CastelnuovoMumford regularity are three important and closely related invariants in commutative algebra and algebraic geometry. For example, if $S=\mathbb{K}\left[x_{1}, \ldots, x_{n}\right]$ is a polynomial ring over a field $\mathbb{K}$ and $I \subset S$ is a monomial ideal, then thanks to Auslander and Buchsbaum [1, Theorem 1.3.3], we know that

$$
\operatorname{depth}(S / I)+\operatorname{proj} \operatorname{dim}(S / I)=n .
$$

If, in addition, $I$ is squarefree, then $I$ has an Alexander dual $I^{\vee}$, which is also a squarefree monomial ideal. Now a result of Terai [25, Corollary 0.3 ] asserts that

$$
\operatorname{proj} \operatorname{dim}(I)=\operatorname{reg}\left(S / I^{\vee}\right)
$$

There are numerous works trying to compute or estimate these three invariants. For instance, Lyubeznik considered the size of monomial ideals in the article [19]. Let $I=\bigcap_{i=1}^{s} Q_{i}$ be an irredundant primary decomposition of a monomial ideal $I$ in $S$, where the $Q_{i}$ 's are also monomial ideals. The size of $I$, denoted by $\operatorname{size}(I)$, is the number $v+n-h-1$, where $v$ is the minimal number $t$ such that there exist $j_{1}<\cdots<j_{t}$ with $\sqrt{\sum_{k=1}^{t} Q_{j_{k}}}=\sqrt{\sum_{j=1}^{s} Q_{j}}$, and where

2010 AMS Mathematics subject classification. Primary 05C65, 05E40.

Keywords and phrases. Squarefree monomial ideal, Stanley depth, Stanley regularity, clutter.

This work was supported by the National Natural Science Foundation of China (No. 11201445) and the Fundamental Research Funds for the Central Universities.

Received by the editors on January 25, 2014. 
$h=\mathrm{ht} \sum_{j=1}^{s} Q_{j}$. Lyubeznik [19, Proposition 2] showed that

$$
\operatorname{depth}(S / I) \geq \operatorname{size}(I),
$$

and consequently,

$$
\operatorname{depth}(I) \geq \operatorname{size}(I)+1 .
$$

A related result, due to Herzog, Popescu and Vladoiu [13, Theorem $3.1]$, asserts that

$$
\operatorname{sdepth}(I) \geq \operatorname{size}(I)+1,
$$

where $\operatorname{sdepth}(I)$ is the Stanley depth of $I$. We will explain the notion of Stanley depth in the next section.

It is conjectured by Stanley [24] that

$$
\operatorname{sdepth}(M) \geq \operatorname{depth}(M)
$$

for all finitely generated $\mathbb{Z}^{n}$-graded $S$-modules. Obviously, Stanley's conjecture (1.4) for $M=I$ with Lyubeznik's result (1.2) implies the inequality (1.3).

It is worth mentioning that squarefree monomial ideals can be naturally related to clutters. Among many others, recent work of Dao and Schweig [6], Hà and Woodroofe [9], Lin and McCullough [18] and Woodroofe [27] provided several very nice bounds for estimating depth and Castelnuovo-Mumford regularity. This research involves considerations of combinatorial data from the clutter associated to the squarefree monomial ideals. Thus, analogous to [13], it is natural to ask whether the above work can find counterparts when estimating Stanley depth and Stanley regularity?

Now it is time to outline the structure of our paper. In Section 2, we provide preliminary background for notions like clutter, filtration depth, Stanley depth and Stanley regularity, respectively.

In Section 3, we will study the method of Dao and Schweig [6], and provide a lower bound of the Stanley depth of squarefree monomials $I$ in terms of the index of edge domination of the associated clutter. If $\mathcal{C}$ is a clutter, a collection $F$ of edges in $\mathcal{C}$ is called edgewise dominant if, for every vertex $v \in V\left(\mathcal{C}^{\text {red }}\right)$ which is not contained in some edge of $F$ or contained in a trivial edge, it has a neighbor contained in some edge 
of $F$. The index of edgewise domination is the number

$$
\epsilon(\mathcal{C})=\min \{|F|: F \subset E(\mathcal{C}) \text { is edgewise dominant }\} .
$$

Our first main result is

Theorem (3.7). Let $\mathcal{C}$ be a clutter and $I(\mathcal{C})$ be the corresponding edge ideal in $S$. Then

$$
\min \{\operatorname{depth}(S / I(\mathcal{C})), \operatorname{sdepth}(S / I(\mathcal{C}))\} \geq \epsilon(\mathcal{C})+n-\left|V\left(\mathcal{C}^{\text {red }}\right)\right| .
$$

In Section 4, we employ the splitting method in Herzog, Popescu and Vladoiu [13] and estimate the Stanley regularity of $S / I$. This work is related to the article [18] by Lin and McCullough. We show that

Theorem (4.1). Let $\mathcal{C}=(V, E)$ be a clutter and $\mathcal{C}^{\prime}=\left(V, E^{\prime}\right)$ the clutter obtained by removing all edges with free vertices from $\mathcal{C}$. Let $\beta\left(\mathcal{C}^{\prime}\right)$ be the matching number of $\mathcal{C}^{\prime}$. Then

$$
\operatorname{sreg}(S / I(\mathcal{C})) \leq|V|-|E|+\left|E^{\prime}\right|-\beta\left(\mathcal{C}^{\prime}\right) .
$$

In Section 5, we will start by establishing a key result that is similar to the Castelnuovo-Mumford regularity version by Kalai and Meshulam. We will apply it to give various upper bounds for the Stanley regularity of $S / I$ in terms of various packing invariants of the associated clutter.

To be more specific, we will study the notion of co-chordal cover number of a simple graph $G$, which is the minimum number of co-chordal subgraphs required to cover $G$. Similar to a result by Woodroofe [27], we assert that

Theorem (5.6). For any simple graph $G$, we have $\operatorname{sreg}(S / I(G)) \leq$ $\operatorname{cochord}(G)$.

Our last result is based on the notion of 2-collage, introduced by Hà and Woodroofe in [9]. Let $\mathcal{C}$ be a clutter. Then a 2-collage for $\mathcal{C}$ is a subset $C$ of edges with the property that for each $E \in E(\mathcal{C})$ we can delete a vertex $v$ so that $E \backslash\{v\}$ is contained in some edge of $C$. We claim that 
Theorem (5.16). If $\left\{E_{1}, \ldots, E_{s}\right\}$ is a 2 -collage in the clutter $\mathcal{C}$, then

$$
\operatorname{sreg}(S / I(\mathcal{C})) \leq \sum_{i=1}^{s}\left(\left|E_{i}\right|-1\right) .
$$

Here is the final comment before we start a new section. Stanley's conjecture (1.4) is still widely open so far. This happens partly due to the lack of powerful tools like the long exact sequence and the depth lemma [1, Proposition 1.2.9]. What we have so far that is most similar to the depth lemma is as follows: let $0 \rightarrow M \rightarrow N \rightarrow L \rightarrow 0$ be a short exact sequence of finitely generated $\mathbb{Z}^{n}$-graded $S$-modules, then

$$
\operatorname{sdepth}(N) \geq \min \{\operatorname{sdepth}(M), \operatorname{sdepth}(L)\}
$$

by [2, Proposition 2.6]. Since the research on depth, projective dimension and Castelnuovo-Mumford regularity depends heavily on applying the depth lemma (or similar results for the other two invariants), it is not a trivial work for establishing parallel results for Stanley depth and Stanley regularity. For instance, it is still conjectured [13] (but not established) that

$$
\operatorname{sdepth}(I) \geq \operatorname{sdepth}(S / I)+1 .
$$

2. Preliminaries. We begin by recalling basic notation and terminology from commutative algebra and combinatorics. For further reading, one can refer to $[\mathbf{1}, \mathbf{1 1}, \mathbf{1 2}, \mathbf{8}]$.

2.1. Clutters. A clutter $\mathcal{C}=(V, E)$ over the vertex set $V(\mathcal{C})=V$ consists of a collection $E(\mathcal{C})=E$ of subsets of $V$, called the edges of $\mathcal{C}$, with the property that no edge contains another. Clutters are also known as simple hypergraphs or Sperner systems. We will only consider clutters whose vertex set is finite.

Two distinct vertices in $V(\mathcal{C})$ are neighbors if there is an edge of $\mathcal{C}$ that contains these vertices. A vertex $v \in V(\mathcal{C})$ is isolated if it does not appear in any edge in $E(\mathcal{C})$. We will write is $(\mathcal{C})$ for the set of isolated vertices and $\mathcal{C}^{\text {red }}$ for the clutter from $\mathcal{C}$ with its isolated vertices removed. 
An edge $e \in E(\mathcal{C})$ is trivial if it contains only one vertex in $V(\mathcal{C})$. Trivial edges are also called isolated loops. When the cardinality of each edge equals a fixed integer $d \geq 2$, the clutter $\mathcal{C}$ is $d$-uniform.

A collection of edges in $\mathcal{C}$ is called a matching if the edges in this collection are pairwise disjoint. The maximum size of a matching in $\mathcal{C}$ is called its matching number. The minimal size of a maximal matching is called the minimax matching number.

For a nonempty subset $A$ of vertices in $\mathcal{C}$, let $\mathcal{C}+A$ denote the clutter whose edges are the minimal sets of $E(\mathcal{C}) \cup\{A\}$ and whose vertex set is still $V(\mathcal{C})$. Meanwhile, let $\mathcal{C}: A$ be the clutter whose edges are the minimal sets of $\{e \backslash A: e \in E(\mathcal{C})\}$ and whose vertex set is $V(\mathcal{C}) \backslash A$.

For simplicity, we often identify vertex sets with subsets of the variables $\{\boldsymbol{x}\}:=\left\{x_{1}, \ldots, x_{n}\right\}$. If $A$ is a subset of $\{\boldsymbol{x}\}$, we write $\boldsymbol{x}^{A}$ for the squarefree monomial $\prod_{x \in A} x$ in $S=\mathbb{K}\left[x_{1}, \ldots, x_{n}\right]$. Thus, the clutter $\mathcal{C}$ corresponds to a squarefree monomial ideal

$$
I(\mathcal{C})=\left\langle\boldsymbol{x}^{e}: e \in E(\mathcal{C})\right\rangle \subset S .
$$

This ideal is called the edge ideal of $\mathcal{C}$. Naturally, the clutters $\mathcal{C}+A$ and $\mathcal{C}: A$ correspond to the squarefree monomial ideals $\left\langle I(\mathcal{C}), \boldsymbol{x}^{A}\right\rangle$ and $I(\mathcal{C}): S \boldsymbol{x}^{A}$, respectively.

2.2. Filtration depth and Stanley depth. Recall that a sequence

$$
\mathcal{F}: 0=M_{0} \subset M_{1} \subset \cdots \subset M_{m}=M
$$

of $\mathbb{Z}^{n}$-graded submodules of $M$ is a prime filtration if each $M_{i} / M_{i-1} \cong$ $\left(S / P_{i}\right)\left(-\boldsymbol{a}_{i}\right)$ for some integral vectors $\boldsymbol{a}_{i} \in \mathbb{Z}^{n}$ and some monomial prime ideals $P_{i}$. The set of the primes $\left\{P_{1}, \ldots, P_{m}\right\}$ is the support of $\mathcal{F}$, which shall be denoted by $\operatorname{supp}(\mathcal{F})$. Now

$$
\operatorname{fdepth}(\mathcal{F}):=\min \{\operatorname{dim}(S / P): P \in \operatorname{supp}(\mathcal{F})\}
$$

is the filtration depth of $\mathcal{F}$ and

$$
\operatorname{fdepth}(M):=\max \{\operatorname{fdepth}(\mathcal{F}): \mathcal{F} \text { is a prime filtration of } M\}
$$

is the filtration depth of $M$.

On the other hand, if $M$ is a nonzero finitely generated $\mathbb{Z}^{n}$-graded $S$-module, $u \in M$ is a homogeneous element and $Z$ is a subset of $\{\boldsymbol{x}\}$, then $u \mathbb{K}[Z]$ is the $\mathbb{K}$-subspace of $M$ generated by all elements $u v$ where 
$v$ is a monomial in $\mathbb{K}[Z]$. A presentation of $M$ as a finite direct sum of such spaces $\mathcal{D}: M=\bigoplus_{i=1}^{r} u_{i} \mathbb{K}\left[Z_{i}\right]$ is called a Stanley decomposition of $M$. Set $\operatorname{sdepth}(\mathcal{D})=\min \left\{\left|Z_{i}\right|: i=1, \ldots, r\right\}$ and $\operatorname{sdepth}(M)=\max \{\operatorname{sdepth}(\mathcal{D}): \mathcal{D}$ is a Stanley decomposition of $M\}$.

We have the following relations among depth, filtration depth and Stanley depth.

Lemma 2.1 ([14, Proposition 1.3]). Let $M$ be a nonzero finitely generated $\mathbb{Z}^{n}$-graded $S$-module. Then

$\operatorname{fdepth}(M) \leq \operatorname{depth}(M), \operatorname{sdepth}(M) \leq \min \{\operatorname{dim}(S / P): P \in \operatorname{Ass}(M)\}$.

Later in this paper, we need the following facts for Stanley depth.

Lemma 2.2. Let $M$ be a nonzero finitely generated $\mathbb{Z}^{n}$-graded $S$ module.

(a) The module $M$ is $S$-free if and only if $\operatorname{sdepth}(M)=n$.

(b) If $\operatorname{sdepth}(M)=0$, then $\operatorname{depth}(M)=0$.

(c) If $\operatorname{depth}(M)=0$ and $\operatorname{dim}_{\mathbb{K}} M_{\boldsymbol{a}} \leq 1$ for all $\boldsymbol{a} \in \mathbb{Z}^{n}$, then $\operatorname{sdepth}(M)=0$.

The proof can be found, for instance, in [2, Proposition 2.13] and [3, Lemma 1.2, Theorem 1.4].

2.3. Stanley regularity. The starting point is Terai's duality theorem via Alexander dual for squarefree monomial ideals. Let $I=$ $\left(x_{11} \cdots x_{1 i_{1}}, \ldots, x_{s 1} \cdots x_{s i_{s}}\right) \subset S=\mathbb{K}\left[x_{1}, \ldots, x_{n}\right]$ be a squarefree monomial ideal. Then the Alexander dual of $I$ is

$$
I^{\vee}=\left(x_{11}, \ldots, x_{1 i_{1}}\right) \cap \cdots \cap\left(x_{s 1}, \ldots, x_{s i_{s}}\right)
$$

with the property that $\left(I^{\vee}\right)^{\vee}=I$. Terai [25, Corollary 0.3] (see also [12, Proposition 8.1.10]) proved that

$$
\operatorname{proj} \operatorname{dim}(I)=\operatorname{reg}\left(S / I^{\vee}\right) .
$$

As established in $[\mathbf{2 2}, \mathbf{2 8}]$, Alexander duality can also be extended to finitely generated squarefree modules. Let $M$ be such a module and 
$\mathcal{D}: M=\bigoplus_{i=1}^{r} u_{i} \mathbb{K}\left[Z_{i}\right]$ a squarefree Stanley decomposition. Then the Stanley regularity of $\mathcal{D}$ is

$$
\operatorname{sreg}(\mathcal{D}):=\max \left\{\operatorname{deg}\left(u_{i}\right): 1 \leq i \leq m\right\}
$$

and the Stanley regularity of $M$ is

$\operatorname{sreg}(M):=\min \{\operatorname{sreg}(\mathcal{D}): \mathcal{D}$ is a Stanley decomposition of $M\}$.

Similar to (1.5), it is straightforward to see that, for a short exact sequence $0 \rightarrow M \rightarrow N \rightarrow L \rightarrow 0$ of finitely generated squarefree modules, we have

$$
\operatorname{sreg}(N) \leq \min \{\operatorname{sreg}(M), \operatorname{sreg}(L)\} .
$$

The following key result plays the same role as the Terai's duality theorem.

Lemma 2.3 ([23, Theorem 3.7], [11, Corollary 46]). If $I$ is a squarefree monomial ideal of $S$, then $\operatorname{sreg}(S / I)=n-\operatorname{sdepth}\left(I^{\vee}\right)$ and $\operatorname{sreg}(I)=n-\operatorname{sdepth}\left(S / I^{\vee}\right)$.

Remark 2.4. Suppose $I$ is a squarefree monomial ideal of $S=$ $\mathbb{K}\left[x_{1}, \ldots, x_{n}\right]$ and $S^{\prime}=S\left[x_{n+1}\right]$. Then $\operatorname{sreg}\left(I S^{\prime}\right)=\operatorname{sreg}(I)$ and $\operatorname{sreg}\left(S^{\prime} /\left(I S^{\prime}\right)\right)=\operatorname{sreg}(S / I)$ by virtue of the above lemma together with [14, Lemma 3.6]. In other words, the isolated vertices are irrelevant for computing the Stanley regularity of edge ideals.

The following inequality is dual to the Stanley's conjecture (1.4).

Conjecture 2.5 ([23]). Let $J \subset I$ be squarefree monomial ideals. Then $\operatorname{sreg}(I / J) \leq \operatorname{reg}(I / J)$.

Remark 2.6. Let $I \subset S$ be a squarefree monomial ideal. Then $\operatorname{sreg}(S / I)=0$ if and only if $I$ is a prime ideal generated by a set of variables. To see this, it suffices to mention that $\operatorname{sdepth}\left(I^{\vee}\right)=n$ if and only if $I^{\vee}$ is principal.

3. Edge domination and Stanley depth. Let $\mathcal{C}$ be a clutter. The following definition is due to [6]. 
Definition 3.1. A collection $F$ of edges in $\mathcal{C}$ is called edgewise dominant if, for every vertex $v \in V\left(\mathcal{C}^{\text {red }}\right)$ which is not contained in some edge of $F$ or contained in a trivial edge, it has a neighbor contained in some edge of $F$. The index of edgewise domination is the number

$$
\epsilon(\mathcal{C})=\min \{|F|: F \subset E(\mathcal{C}) \text { is edgewise dominant }\} .
$$

Dao and Schweig [6, Theorem 3.2] proved that $\operatorname{proj} \operatorname{dim}(S / I(\mathcal{C})) \leq$ $\left|V\left(\mathcal{C}^{\text {red }}\right)\right|-\epsilon\left(\mathcal{C}^{\text {red }}\right)$. This result, by a theorem of Auslander and Buchsbaum [1, Theorem 1.3.3], is equivalent to saying that

$$
\operatorname{depth}(S / I(\mathcal{C})) \geq \epsilon(\mathcal{C})+n-\left|V\left(\mathcal{C}^{\text {red }}\right)\right| .
$$

It is clear that Stanley's conjecture (1.4) for $M=S / I(\mathcal{C})$ with the above inequality implies that

$$
\operatorname{sdepth}(S / I(\mathcal{C})) \geq \epsilon(\mathcal{C})+n-\left|V\left(\mathcal{C}^{\text {red }}\right)\right| .
$$

This is the result that we want to establish in this section. To this end, let us go over some basic concepts and constructions from the original paper [6].

Definition 3.2. A collection $\Phi$ of clutters is hereditary if, for any clutter $\mathcal{C} \in \Phi$ and any nonempty subset $A$ of vertices of $\mathcal{C}$, the clutters $\mathcal{C}: A, \mathcal{C}+A$ and $\mathcal{C}^{\text {red }}$ are all in $\Phi$.

Suppose $\Phi$ is a hereditary collection of clutters and $f: \Phi \rightarrow \mathbb{N}$ is a function. We consider the following conditions for $f$.

(DS.1) $f(\mathcal{C}) \leq \max \{f(\mathcal{C}+A), f(\mathcal{C}: A)\}$ for all $\mathcal{C} \in \Phi$ and nonempty $A \subset V(\mathcal{C})$.

(DS.2) $f\left(\mathcal{C}^{\text {red }}\right)=f(\mathcal{C})$ for all $\mathcal{C} \in \Phi$.

$($ DS.3) $f(\mathcal{C}) \leq|V(\mathcal{C})|$ when $E(\mathcal{C})=\emptyset$.

(DS.4) $f(\mathcal{C})=0$ when $E(\mathcal{C})=\emptyset$.

(DS.5) $f(\mathcal{C})=0$ when $\mathcal{C}$ has only trivial edges.

(DS.6) $f(\mathcal{C}) \leq|V(\mathcal{C})|$ when $\mathcal{C}$ has only trivial edges.

(DS.7) For any $\mathcal{C} \in \Phi$ with at least one non-trivial edge, there exists a sequence of nonempty subsets $A_{1}, \ldots, A_{t}$ of $V(\mathcal{C})$ such that, for the clutters $\mathcal{C}_{i}:=\mathcal{C}+\sum_{j=1}^{i} A_{j}$, the following properties are satisfied:

- $\mid$ is $\left(\mathcal{C}_{t}\right) \mid>0$ and $f\left(\mathcal{C}_{t}\right)+\mid$ is $\left(\mathcal{C}_{t}\right) \mid \geq f(\mathcal{C})$, and 
- for each $i, f\left(\mathcal{C}_{i-1}: A_{i}\right)+\mid$ is $\left(\mathcal{C}_{i-1}: A_{i}\right)|+| A_{i} \mid \geq f(\mathcal{C})$.

Observation 3.3. When $g(\mathcal{C})=\operatorname{proj} \operatorname{dim}\left(\mathbb{K}\left[x_{1}, \ldots, x_{n}\right] / I(\mathcal{C})\right)$ for clutters $\mathcal{C}$ with $V(\mathcal{C})=\left\{x_{1}, \ldots, x_{n}\right\}$, it is clear that $g$ satisfies the above conditions (DS.1), (DS.2), (DS.4) and (DS.6). $3.3]$.

The following key lemma with its proof is adapted from [6, Lemma

Lemma 3.4. Let $\Phi$ be a hereditary class of clutters and $f, g: \Phi \rightarrow \mathbb{N}$ two functions such that

(a) $f$ satisfies conditions (DS.2), (DS.3), (DS.5) and (DS.7), and

(b) $g$ satisfies conditions (DS.1), (DS.2), (DS.4) and (DS.6).

Then, for any $\mathcal{C} \in \Phi, f(\mathcal{C})+g(\mathcal{C}) \leq\left|V\left(\mathcal{C}^{\text {red }}\right)\right|$.

Proof. We prove by induction on $|V(\mathcal{C})|$. Because of condition (DS.2), we may assume that $\mathcal{C}=\mathcal{C}^{\text {red }}$ is a clutter without any isolated vertex. Meanwhile, by conditions (DS.3), (DS.4), (DS.5) and (DS.6), we may further assume that $\mathcal{C}$ has at least one non-trivial edge. Therefore, there exists a sequence of sets $A_{1}, \ldots, A_{t}$ as in condition (DS.7) for the function $f$.

By condition (DS.1) for the function $g$, we are reduced to the following two cases.

(a) $g(\mathcal{C}) \leq g\left(\mathcal{C}_{1}\right) \leq \cdots \leq g\left(\mathcal{C}_{t}\right)=g\left(\mathcal{C}_{t}{ }^{\text {red }}\right)$. Since $\mid$ is $\left(\mathcal{C}_{t}\right) \mid>0$, by induction hypothesis, we have

$$
\begin{aligned}
g(\mathcal{C}) & \leq g\left(\mathcal{C}_{t}{ }^{\text {red }}\right) \leq\left|V\left(\mathcal{C}_{t}{ }^{\text {red }}\right)\right|-f\left(\mathcal{C}_{t}\right) \\
& \leq\left(|V(\mathcal{C})|-\mid \text { is }\left(\mathcal{C}_{t}\right) \mid\right)-\left(f(\mathcal{C})-\mid \text { is }\left(\mathcal{C}_{t}\right) \mid\right) \\
& =|V(\mathcal{C})|-f(\mathcal{C})
\end{aligned}
$$

(b) $g(\mathcal{C}) \leq g\left(\mathcal{C}_{1}\right) \leq \cdots g\left(\mathcal{C}_{i-1}\right) \leq g\left(\mathcal{C}_{i-1}\right.$ : $A_{i}$ ) for some integer $i \in\{1,2, \ldots, t\}$. Since

$$
\left|V\left(\mathcal{C}_{i-1}: A_{i}\right)\right|=\left|V\left(\mathcal{C}_{i-1}\right)\right|-\left|A_{i}\right|<\left|V\left(\mathcal{C}_{i-1}\right)\right|=|V(\mathcal{C})|,
$$


we can apply the induction hypothesis and get

$$
\begin{aligned}
g(\mathcal{C}) \leq & g\left(\mathcal{C}_{i-1}: A\right) \leq\left|V\left(\left(\mathcal{C}_{i-1}: A_{i}\right)^{\mathrm{red}}\right)\right|-f\left(\mathcal{C}_{i-1}: A_{i}\right) \\
\leq & \left(\left|V\left(\mathcal{C}_{i-1}: A_{i}\right)\right|-\mid \text { is }\left(\mathcal{C}_{i-1}: A_{i}\right) \mid\right)-(f(\mathcal{C}) \\
& \left.-\mid \text { is }\left(\mathcal{C}_{i-1}: A_{i}\right)|-| A_{i} \mid\right) \\
= & \left(\left|V\left(\mathcal{C}_{i-1}\right)\right|-\left|A_{i}\right|\right)-f(\mathcal{C})+\left|A_{i}\right|=|V(\mathcal{C})|-f(\mathcal{C}) .
\end{aligned}
$$

Lemma 3.5. Let $\Phi$ be the collection of all clutters whose vertex set is a subset of $\left\{x_{1}, \ldots, x_{n}\right\}$. Then the index of edgewise dominant $\epsilon$ is a function that satisfies conditions (DS.2), (DS.3), (DS.5) and (DS.7).

Proof. It follows easily from the definition that $\epsilon$ satisfies conditions (DS.2), (DS.3) and (DS.5). As for condition (DS.7), let $\mathcal{C}$ be a clutter with at least one non-trivial edge. Let $x$ be a vertex in such an edge, and let $y_{1}, \ldots, y_{t}$ be the neighbors of $x$. If we take $A_{i}=\left\{y_{i}\right\}$, then the proof of [6, Theorem 3.2] shows that $\epsilon$ satisfies condition (DS.7), which we will not repeat here.

Here is the main result of this section.

Theorem 3.6. Let $\mathcal{C}$ be a clutter and $I(\mathcal{C})$ the corresponding edge ideal in $S$. Then

$$
\operatorname{fdepth}(S / I(\mathcal{C})) \geq \epsilon(\mathcal{C})+n-\left|V\left(\mathcal{C}^{\text {red }}\right)\right|
$$

Proof. Let $\Phi$ be the collection of all clutters whose vertex set is a subset of $\left\{x_{1}, \ldots, x_{n}\right\}$. It suffices to show that $g(\mathcal{C}):=n-$ fdepth $(S / I(\mathcal{C}))$ is a function from $\Phi$ to $\mathbb{N}$ that satisfies conditions (DS.1), (DS.2), (DS.4) and (DS.6).

(a) $\operatorname{Since} \operatorname{fdepth}(S / I(\mathcal{C}))$ takes value in $\{0,1, \ldots, n=\operatorname{dim}(S)\}, g$ is a function from $\Phi$ to $\mathbb{N}$.

(b) Let $\mathcal{C}$ be a clutter in $\Phi$ and $A \subset V(\mathcal{C})$. There is a natural short exact sequence

$$
0 \rightarrow S /\left(I(\mathcal{C}): \boldsymbol{x}^{A}\right) \rightarrow S / I(\mathcal{C}) \rightarrow S /\left(I(\mathcal{C}), \boldsymbol{x}^{A}\right) \rightarrow 0 .
$$

Obviously, a prime filtration of $S /\left(I(\mathcal{C}): \boldsymbol{x}^{A}\right)$ can be combined with a prime filtration of $S /\left(I(\mathcal{C}), \boldsymbol{x}^{A}\right)$ to yield a prime filtration 
of $S / I(\mathcal{C})$. Hence,

$$
\begin{aligned}
& \operatorname{fdepth}(S / I(\mathcal{C})) \\
& \quad \geq \min \left\{\operatorname{fdepth}\left(S /\left(I(\mathcal{C}): \boldsymbol{x}^{A}\right), \operatorname{fdepth}\left(S /\left(I(\mathcal{C}), \boldsymbol{x}^{A}\right)\right)\right)\right\},
\end{aligned}
$$

and $g$ satisfies condition (DS.1).

(c) Since $I(\mathcal{C})=I\left(\mathcal{C}^{\text {red }}\right)$ in $S$, the function $g$ satisfies condition (DS.2).

(d) If $\mathcal{C} \in \Phi$ with $E(\mathcal{C})=\emptyset$, then $I(\mathcal{C})=0$. Hence, $g(\mathcal{C})=$ $n-\operatorname{fdepth}(S)=0$ and satisfies condition (DS.4).

(e) If $\mathcal{C} \in \Phi$ has only trivial edges, $I(\mathcal{C})=\langle y: y \in V(\mathcal{C})\rangle$. Thus fdepth $(S / I(\mathcal{C}))=n-|V(\mathcal{C})|$ and $g$ satisfies condition (DS.6).

Notice that the above result is slightly stronger than [6, Theorem $3.2]$ :

Theorem 3.7. Let $\mathcal{C}$ be a clutter and $I(\mathcal{C})$ the corresponding edge ideal in $S$. Then

$$
\min \{\operatorname{depth}(S / I(\mathcal{C})), \operatorname{sdepth}(S / I(\mathcal{C}))\} \geq \epsilon(\mathcal{C})+n-\left|V\left(\mathcal{C}^{\text {red }}\right)\right| .
$$

Proof. Since

$$
\operatorname{fdepth}(M) \leq \min \{\operatorname{depth}(M), \operatorname{sdepth}(M)\},
$$

by Lemma 2.1, this result follows from Theorem 3.6 immediately.

In particular, we get the nice lower bound (3.2) for the Stanley depth of $S / I(\mathcal{C})$, as expected.

Corollary 3.8. Let $\mathcal{C}$ be a clutter and $I(\mathcal{C})$ the corresponding edge ideal in $S$. Then there is a prime filtration of $S / I(\mathcal{C})$

$$
\mathcal{F}: 0=M_{0} \subset M_{1} \subset \cdots \subset M_{m}=M
$$

such that, for each $i, M_{i} / M_{i-1} \cong\left(S / P_{i}\right)\left(-\boldsymbol{a}_{i}\right)$ is of dimension at least $\epsilon(\mathcal{C})+n-\left|V\left(\mathcal{C}^{\text {red }}\right)\right|$ and $\boldsymbol{a}_{i}$ is a squarefree vector in $\mathbb{N}^{n}$.

Proof. As pointed out in [14, Corollary 2.5], the filtration depth of $S / I(\mathcal{C})$ can be computed by checking special partitions of the poset $P_{S / I(\mathcal{C})}^{\mathbf{1}}$ whose elements are the squarefree monomials in $S \backslash I(\mathcal{C})$. Since 
$\operatorname{fdepth}(S / I(\mathcal{C})) \geq \epsilon(\mathcal{C})+n-\left|V\left(\mathcal{C}^{\text {red }}\right)\right|$ by Theorem 3.6, the expected filtration exists by virtue of [14, Theorem 2.4 (a)].

4. Splitting and Stanley regularity. If $I$ is a squarefree monomial ideal minimally generated by monomials $u_{1}, \ldots, u_{m}$ and $w$ is the smallest number $t$ such that there exists integers $1 \leq i_{1}<i_{2}<\cdots<$ $i_{t} \leq m$ such that

$$
\operatorname{lcm}\left(u_{i_{1}}, u_{i_{2}}, \ldots, u_{i_{t}}\right)=\operatorname{lcm}\left(u_{1}, u_{2}, \ldots, u_{m}\right),
$$

then the number $\operatorname{deg} \operatorname{lcm}\left(u_{1}, \ldots, u_{m}\right)-w$ is called the cosize of $I$, denoted by $\operatorname{cosize}(I)$. Now, due to inequality (1.3), we have

$$
\operatorname{sreg}(S / I(\mathcal{C})) \leq \operatorname{cosize}(I(\mathcal{C}))
$$

by [13, Corollary 3.4].

Suppose in the above setting each monomial $u_{i}$ contains a free variable, i.e., a variable that divides this $u_{i}$ but not any other monomial generator. Then the Taylor resolution of $S / I$ is minimal by [18, Proposition 4.1] and $\operatorname{reg}(S / I)$ is exactly the number $|X|-m$ where $X$ is the set of variables showing in these $u_{i}$ 's. Due to Conjecture 2.5, it is natural to ask whether the inequality

$$
\operatorname{sreg}(S / I) \leq|X|-m
$$

holds in general. As a matter of fact, the inequality (4.2) holds as a special case of the inequality (4.1) by recognizing that $\operatorname{cosize}(I)=$ $|X|-m$. On the other hand, equality does not hold for (4.2) in general. For instance, when $I=\left\langle x_{1} x_{2} \cdots x_{n}\right\rangle$ is a principal ideal, then $\operatorname{sreg}(S / I)=\lfloor n / 2\rfloor<n-1$ for $n \geq 3$.

Vertices of a clutter $\mathcal{C}$ that correspond to the free variables for its edges ideal $I(\mathcal{C})$ are also free, i.e., an edge $e \in E(\mathcal{C})$ is said to contain a free vertex if there exists some vertex $x \in e$ such that $x$ does not belong to any other edges in $\mathcal{C}$. The subsequent generalization of the inequality (4.2) is parallel to [18, Theorem 4.9]. We adopt the following version, rephrased by [8, Theorem 4.20].

Theorem 4.1. Let $\mathcal{C}=(V, E)$ be a clutter and $\mathcal{C}^{\prime}=\left(V, E^{\prime}\right)$ the clutter obtained by removing all edges with free vertices from $\mathcal{C}$. Let $\beta\left(\mathcal{C}^{\prime}\right)$ be 
the matching number of $\mathcal{C}^{\prime}$. Then

$$
\operatorname{sreg}(S / I(\mathcal{C})) \leq|V|-|E|+\left|E^{\prime}\right|-\beta\left(\mathcal{C}^{\prime}\right)
$$

Since our proof for Theorem 4.1 and Lemma 5.15 depends heavily on the splitting method in [13], we will outline the key ingredients here.

Construction 4.2. Let $I=\bigcap_{i=1}^{s} P_{i}$ be an irredundant primary decomposition of the squarefree monomial ideal $I$ in $S$. All the $P_{i}$ 's are necessarily generated by subsets of $\left\{x_{1}, \ldots, x_{n}\right\}$. We will take the variables in some specific $P_{i}$ as a splitting set. Without loss of generality, we may choose $P_{1}$ and assume that $P_{1}=\left\langle x_{1}, x_{2}, \ldots, x_{r}\right\rangle$. Write $S^{\prime}=\mathbb{K}\left[x_{1}, \ldots, x_{r}\right]$ and $S^{\prime \prime}=\mathbb{K}\left[x_{r+1}, \ldots, x_{n}\right]$. For each $P_{i}$, let $P_{i}^{\prime}=P_{i} \cap S^{\prime}$ and $P_{i}^{\prime \prime}=P_{i} \cap S^{\prime \prime}$. Now, for each subset $\tau \subset[s]:=$ $\{1,2, \ldots, s\}$, let $I_{\tau}$ be the $\mathbb{Z}^{n}$-graded $\mathbb{K}$-vector space spanned by the set of monomials of the form $w=u v$, where $u \in S^{\prime}$ and $v \in S^{\prime \prime}$ are monomials with $u \in \bigcap_{j \notin \tau} P_{j} \backslash \sum_{j \in \tau} P_{j}$ and $v \in \bigcap_{j \in \tau} P_{j}$. Thus, by [13, Proposition 2.1], $I=\bigoplus_{\tau \subset[s]} I_{\tau}$ is a decomposition of $I$ as a direct sum of $\mathbb{Z}^{n}$-graded $\mathbb{K}$-subspaces of $I$ with $I_{[s]}=0$. By the explanation after [13, Proposition 2.1], we can write $I_{\tau}$ as $I_{\tau}=H_{\tau} \otimes_{\mathbb{K}} L_{\tau}$, where

$$
H_{\tau}=\frac{\bigcap_{j \notin \tau} P_{j}^{\prime}+\sum_{j \in \tau} P_{j}^{\prime}}{\sum_{j \in \tau} P_{j}^{\prime}}
$$

and $L_{\tau}=\bigcap_{j \in \tau} P_{j}^{\prime \prime}$.

Proof of Theorem 4.1. Without loss of generality, we may assume that edges $e_{1}, \ldots, e_{a}$ are removed from $\mathcal{C}$ to get the clutter $\mathcal{C}^{\prime}$. We also assume that $\beta\left(\mathcal{C}^{\prime}\right)=b$ such that edges $e_{a+1}, \ldots, e_{a+b}$ form a maximal matching in $\mathcal{C}^{\prime}$. Let the remaining edges be $e_{a+b+1}, \ldots, e_{a+b+c}$. Since we can also assume that $\mathcal{C}$ contains no isolated vertices, we are reduced to proving that

$$
\operatorname{sreg}(S / I(\mathcal{C})) \leq n-(a+b+c)+(b+c)-b=n-a-b .
$$

For each edge $e_{i}$ of $\mathcal{C}$, there is a corresponding monomial prime ideal $P_{i}=\left\langle x_{j}: x_{j} \in e_{i}\right\rangle \subset S$. Now $I(\mathcal{C})^{\vee}=\bigcap_{i=1}^{s} P_{i}$. Since $\mathcal{C}$ contains no 
isolated vertices, $\sum_{i=1}^{s} P_{i}=\left\langle x_{1}, \ldots, x_{n}\right\rangle$. We need to prove that

$$
\operatorname{sdepth}\left(\bigcap_{i=1}^{a+b+c} P_{i}\right) \geq a+b \text {. }
$$

We will prove by induction on the number $a+b+c$. When $a+b+c=1$, this is trivial. When $b=0, \mathcal{C}^{\prime}$ has no edge and all the edges of $\mathcal{C}$ contain free vertices. In this situation, $\operatorname{size}\left(I(\mathcal{C})^{\vee}\right)=a-1$. Thus we are done, thanks to the inequality (1.3).

In the following, we consider the case when $b \geq 1$ and assume that (4.4) holds for smaller $a+b+c$. We will split using the variables in $P_{a+1}$ as in Construction 4.2 and define the rings $S^{\prime}$ and $S^{\prime \prime}$ accordingly. For each $k=1, \ldots, a$, we may assume that $x_{i_{k}}$ is a free vertex in $e_{k}$. Necessarily, $x_{i_{k}} \in P_{k}^{\prime \prime}$. Another key observation is that $P_{i}^{\prime}=0$ for $i=a+2, \ldots, a+b$.

The dual ideal $I(\mathcal{C})^{\vee}$ has a $\mathbb{Z}^{n}$-graded $\mathbb{K}$-subspace decomposition $I(\mathcal{C})^{\vee}=\bigoplus_{\tau \subset[a+b+c]} I_{\tau}$ with $I_{[a+b+c]}=0$. It suffices to consider the case when $I_{\tau}=H_{\tau} \otimes_{\mathbb{K}} L_{\tau} \neq 0$; whence $H_{\tau} \neq 0$ and $L_{\tau} \neq 0$. But for $H_{\tau} \neq 0$ in the presentation (4.3), we need $a+1 \notin \tau$ and $i \in \tau$ for $i=a+2, \ldots, a+b$. Notice that $H_{\tau}$ is isomorphic to a squarefree monomial ideal in $\mathbb{K}\left[x_{i} \mid x_{i} \in P_{1} \backslash \sum_{j \in \tau} P_{j}\right]$. Thus, when $H_{\tau}$ is nonzero, $\operatorname{sdepth}_{S^{\prime}}\left(H_{\tau}\right) \geq 1$ by Lemma 2.2 .

Next, we demonstrate that $\operatorname{sdepth}\left(L_{\tau}\right) \geq a+b-1$ when $L_{\tau} \neq 0$. Notice that $L_{\tau}=\bigcap_{j \in \tau} P_{j}^{\prime \prime}$ with $\{a+2, \ldots, a+b\} \subset \tau$. Suppose that the intersection $L_{\tau}=\bigcap_{j \in \tau^{\prime}} P_{j}^{\prime \prime}$ is an irredundant primary decomposition of $L_{\tau}$. Obviously, $\tau^{\prime}$ is a non-empty subset of $\tau$. For each $i \in\{a+2, \ldots, a+b\}$, if $i \in \tau \backslash \tau^{\prime}$, there must exist some $P_{k_{i}}^{\prime \prime}$ with $P_{k_{i}}^{\prime \prime} \subset P_{i}^{\prime \prime}$ and $k_{i} \in \tau^{\prime}$. Since $P_{1}^{\prime \prime}, \ldots, P_{a}^{\prime \prime}$ all contain free variables while $e_{a+1}, \ldots, a_{a+b}$ are pairwise disjoint, this $k_{i} \in \tau \cap\{a+b+1, \ldots, a+b+c\}$ and $P_{k_{i}}^{\prime \prime}$ is contained in at most one such $P_{j}^{\prime \prime}$ when we limit $j$ to $\{a+2, \ldots, a+b\}$.

Let us check the clutter $\widehat{\mathcal{C}}$ containing edges corresponding to the prime ideals $P_{i}^{\prime \prime}$ with $i \in \tau^{\prime}$. For each $i \in\{1, \ldots, a\} \cap \tau^{\prime}$, let $\widehat{e}_{i}$ be the corresponding edge. This $\widehat{e}_{i}$ still contains a free vertex. For each $i \in\{a+2, \ldots, a+b\}$, if $i \in \tau^{\prime}$, let $\widehat{e}_{i}$ be the edge corresponding to $P_{i}^{\prime \prime}$; otherwise, let $\widehat{e}_{i}$ be the edge corresponding to $P_{k_{i}}^{\prime \prime}$. The edges $\widehat{e}_{a+2}, \ldots, \widehat{e}_{a+b}$ are pairwise disjoint. Thus, by induction hypothesis, we 
have

$\operatorname{sdepth}_{S^{\prime \prime}}\left(L_{\tau}\right) \geq\left(n-\operatorname{ht}\left(P_{a+1}\right)\right)-\mathrm{ht}\left(\sum_{j \in \tau^{\prime}} P_{j}^{\prime \prime}\right)+\left|\tau^{\prime} \cap\{1, \ldots, a\}\right|+(b-1)$.

When $k \in\{1, \ldots, a\} \backslash \tau^{\prime}, x_{i_{k}} \in S^{\prime \prime} \backslash \sum_{j \in \tau^{\prime}} P_{j}^{\prime \prime}$. Hence,

$$
n-\operatorname{ht}\left(P_{a+1}\right)-\operatorname{ht}\left(\sum_{j \in \tau} P_{j}^{\prime \prime}\right) \geq\left|\{1, \ldots, a\} \backslash \tau^{\prime}\right| .
$$

Consequently,

$\operatorname{sdepth}\left(L_{\tau}\right) \geq\left|\{1, \ldots, a\} \backslash \tau^{\prime}\right|+\left|\tau^{\prime} \cap\{1, \ldots, a\}\right|+(b-1)=a+b-1$.

Now

$\operatorname{sdepth}\left(I_{\tau}\right) \geq \operatorname{sdepth}\left(H_{\tau}\right)+\operatorname{sdepth}\left(L_{\tau}\right) \geq 1+a+b-1=a+b$,

as expected. Finally, we arrive at the desired inequality

$\operatorname{sdepth}_{S}\left(I^{\vee}\right) \geq \min \left\{\operatorname{sdepth}_{S}\left(I_{\tau}\right): \tau \subsetneq[a+b+c]\right.$ and $\left.I_{\tau} \neq 0\right\} \geq a+b$.

5. Packing and Stanley regularity. Let $I_{1}, \ldots, I_{s}$ be squarefree monomial ideals in $S$. In [16], Kalai and Meshulam obtained the following results:

(a) $\operatorname{reg}\left(S / \sum_{i=1}^{s} I_{i}\right) \leq \sum_{i=1}^{s} \operatorname{reg}\left(S / I_{i}\right)$, and

(b) $\operatorname{reg}\left(\bigcap_{i=1}^{s} I_{i}\right) \leq \sum_{i=1}^{s} \operatorname{reg}\left(I_{i}\right)$.

These results were later extended to arbitrary (not necessarily squarefree) monomial ideals by Herzog [10]. Since the above inequalities play an indispensable role in the research of $[\mathbf{2 7}, \mathbf{9}]$, we will start by generalizing these results to the Stanley regularity of squarefree monomial ideals.

Lemma 5.1. Let $I_{1}, \ldots, I_{s}$ be squarefree monomial ideals in $S=$ $\mathbb{K}\left[x_{1}, \ldots, x_{n}\right]$. Then

$$
\left(\sum_{i=1}^{s} I_{i}\right)^{\vee}=\bigcap_{i=1}^{s} I_{i}^{\vee} \text { and }\left(\bigcap_{i=1}^{s} I_{i}\right)^{\vee}=\sum_{i=1}^{s} I_{i}^{\vee}
$$


Proof. The first equality follows from definition. The second equality follows from the first one by using the duality $\left(I^{\vee}\right)^{\vee}=I$.

Lemma 5.2. Let $I_{1}, \ldots, I_{s}$ be squarefree monomial ideals in $S=$ $\mathbb{K}\left[x_{1}, \ldots, x_{n}\right]$. Then

(a) $\operatorname{sreg}\left(S / \sum_{i=1}^{s} I_{i}\right) \leq \sum_{i=1}^{s} \operatorname{sreg}\left(S / I_{i}\right)$, and

(b) $\operatorname{sreg}\left(\bigcap_{i=1}^{s} I_{i}\right) \leq \sum_{i=1}^{s} \operatorname{sreg}\left(I_{i}\right)$.

Proof. (a) We have

$\operatorname{sreg}\left(S / \sum_{i=1}^{s} I_{i}\right)=n-\operatorname{sdepth}\left(\sum_{i=1}^{s} I_{i}\right)^{\vee}=n-\operatorname{sdepth}\left(\bigcap_{i=1}^{s} I_{i}^{\vee}\right)$

$$
\begin{aligned}
& \stackrel{(*)}{\leq} n-\left(\sum_{i=1}^{s} \operatorname{sdepth}\left(I_{i}^{\vee}\right)-(s-1) n\right) \\
& =\sum_{i=1}^{s}\left(n-\operatorname{sdepth}\left(I_{i}^{\vee}\right)\right)=\sum_{i=1}^{s} \operatorname{sreg}\left(S / I_{i}\right) .
\end{aligned}
$$

For inequality $(*)$ above, we have applied [4, Corollary $2.11(1)$ ].

(b) This result can be similarly proved by applying [4, Corollary $2.11(4)]$.

5.1. Simple graphs. In this subsection, we will restrict ourselves to the simple graphs, namely, those clutters whose edges all contain exactly two distinct vertices. We will in general denote such a simple graph by $G$ instead of $\mathcal{C}$. And $\bar{G}$ shall be the complement graph of $G$.

Corollary 5.3. If $G=m K_{2}$ is the simple graph of $m$ disjoint edges, then $\operatorname{sreg}(S / I(G))=\lceil m / 2\rceil$.

Proof. It is straightforward to see that the claim holds when $m=1$ and $m=2$. Thus, after partitioning $m K_{2}=2 K_{2}+\cdots+2 K_{2}$ when $m$ is even and $m K_{2}=2 K_{2}+\cdots+2 K_{2}+K_{1}$ when $m$ is odd, we get $\operatorname{sreg}\left(S / I\left(m K_{2}\right)\right) \leq\lceil m / 2\rceil$ by Lemma $5.2(\mathrm{a})$.

On the other hand, we may assume that $G$ contains no isolated vertex. Now, all squarefree monomials in $I\left(m K_{2}\right)^{\vee}$ have degree at least $m$. It is easy to see that there are exactly $2^{m}$ of them having degree $m$ and $m 2^{m-1}$ of them having degree $m+1$. Thus, 
$\operatorname{sdepth}\left(I\left(m K_{2}\right)^{\vee}\right) \leq m+\left\lfloor m 2^{m-1} / 2^{m}\right\rfloor=m+\lfloor m / 2\rfloor$ by [15, Lemma 2.4]. Thus, $\operatorname{sreg}\left(S / I\left(m K_{2}\right)\right) \geq 2 m-(m+\lfloor m / 2\rfloor)=\lceil m / 2\rceil$.

If $A \subset V(G)$, then $G \backslash A$ denotes the induced subgraph on $V(G) \backslash A$. When $A=\left\{x_{v}\right\}$ consists of exactly one vertex, we will write $G \backslash x_{v}$ instead of $G \backslash A$.

A clique of $G$ is a subset of pairwise adjacent vertices. Cliques are not required to be maximal. Now, for a vertex $x \in V(G)$, the set of neighbors of $x$ is given by

$$
N(x)=\{y \in V(G) \mid\{x, y\} \in E(G)\} .
$$

The vertex $x$ is simplicial if $N(x)$ induces a clique in $G$.

Lemma 5.4. Let $G$ be a simple graph with $V(G)=\left\{x_{1}, \ldots, x_{n}\right\}$ and $x_{v}$ a simplicial vertex of $G$. Let $G_{1}=G \backslash x_{v}$ and $I=I(\bar{G}), J=I\left(\overline{G_{1}}\right)$ be the corresponding edge ideals in $S=\mathbb{K}\left[x_{1}, \ldots, x_{n}\right]$.

(a) If $J \neq 0$, then $\operatorname{sreg}(S / I) \leq \operatorname{sreg}(S / J)$.

(b) If $J=0$, then $\operatorname{sreg}(S / I)=1$.

Proof.

(a) Suppose $J \neq 0$. We will follow the strategy of [20, Theorem 2.7]. By Lemma 2.3, it suffices to $\operatorname{show}$ that $\operatorname{sdepth}\left(I^{\vee}\right) \geq$ $\operatorname{sdepth}\left(J^{\vee}\right)$. We may assume that $N\left(x_{v}\right)=\left\{x_{1}, \ldots, x_{v-1}\right\}$ and the minimal monomial generators of $J$ belong to $\mathbb{K}\left[x_{1}, \ldots\right.$, $\left.\widehat{x_{v}}, \ldots, x_{n}\right]$. Now $I=J+\left\langle x_{v} x_{i}: v<i \leq n\right\rangle$. Since $x_{v}$ is a simplicial vertex of $G$, we have $x_{v+1} \cdots x_{n} \in J^{\vee}$. Now,

$$
\begin{aligned}
I^{\vee} & =J^{\vee} \cap\left(\bigcap_{i=v+1}^{n}\left\langle x_{v}, x_{i}\right\rangle\right) \\
& =J^{\vee} \cap\left(x_{v}, x_{v+1} \cdots x_{n}\right) \\
& =\left(\left\langle x_{v}\right\rangle \cap J^{\vee}\right)+\left\langle x_{v+1} \cdots x_{n}\right\rangle
\end{aligned}
$$

with the property that $\left(\left\langle x_{v}\right\rangle \cap J^{\vee}\right) \cap\left\langle x_{v+1} \cdots x_{n}\right\rangle=\left\langle x_{v} x_{v+1} \cdots x_{n}\right\rangle$ and $\left\langle x_{v}\right\rangle \cap J^{\vee}=x_{v} J^{\vee}$. Therefore, by [2, Proposition 2.6], we 
have

$$
\begin{aligned}
\operatorname{sdepth}\left(I^{\vee}\right) \geq & \min \left\{\operatorname{sdepth}\left(x_{v} J^{\vee}\right),\right. \\
& \left.\quad \operatorname{sdepth}\left(\left\langle x_{v+1} \cdots x_{n}\right\rangle /\left\langle x_{v} \cdots x_{n}\right\rangle\right)\right\} \\
= & \min \left\{\operatorname{sdepth}\left(J^{\vee}\right), \operatorname{sdepth}\left(S /\left\langle x_{v}\right\rangle\right)\right\} \\
= & \min \left\{\operatorname{sdepth}\left(J^{\vee}\right), n-1\right\} \\
= & \operatorname{sdepth}\left(J^{\vee}\right) .
\end{aligned}
$$

Notice that $\operatorname{sdepth}\left(J^{\vee}\right)=n$ if and only if $J^{\vee}$ is principal, whence $J$ is a prime ideal generated by a set of variables. But this cannot happen for the edge ideal of a finite simple graph, unless $J=0$.

(b) Suppose $J=0$. We might assume that $x_{v}=x_{1}$ and $I=$ $x_{1}\left\langle x_{2}, \ldots, x_{n}\right\rangle$. Now $I^{\vee}=\left\langle x_{1}, x_{2} \cdots x_{n}\right\rangle$ is two-generated; thus, $\operatorname{sdepth}\left(I^{\vee}\right)=n-1$ by $[\mathbf{1 4}$, Corollary 3.5]. It follows that $\operatorname{sreg}(I)=1$.

A graph $G$ is chordal if every induced cycle in $G$ has length 3 and is co-chordal if the complement graph $\bar{G}$ is chordal. It follows from Fröberg's classification of edge ideals with linear resolutions [14] that $\operatorname{reg}(R / I(G)) \leq 1$ if and only if $\bar{G}$ is co-chordal. Due to Conjecture 2.5, it is natural to prove the following result that is partially parallel to Fröberg's classification.

Theorem 5.5. If $G$ is a co-chordal graph with at least one edge, then $\operatorname{sreg}(S / I(G)) \leq 1$.

Proof. As observed by [20, Theorem 2.8], the paper [7] has actually shown that a simple graph is chordal if and only if every induced subgraph of it has a simplicial vertex. Thus, we use a induction on the number of vertices of the complement graph $\bar{G}$ and apply Lemma 5.4.

The co-chordal cover number, denoted by cochord $(G)$, is the minimum number of co-chordal subgraphs required to cover the edges of $G$. Like [27, Lemma 1], we have the following result

Theorem 5.6. For any simple graph $G$, we have $\operatorname{sreg}(S / I(G)) \leq$ $\operatorname{cochord}(G)$. 
Proof. We cover the graph $G$ by co-chordal subgraphs $G_{1}, \ldots, G_{c}$ where $c=\operatorname{cochord}(G)$ and let $I_{i}=I\left(G_{i}\right)$. Now the result follows directly from Theorem 5.5 and Lemma 5.2 (a).

An independent set of $G$ is a subset of pairwise non-adjacent vertices. And $G$ is a split graph if $V(G)$ can be partitioned into a clique and an (induced) independent set. Split graphs are both chordal and cochordal. Covering the edges of $G$ with split graphs allows us to have

Corollary 5.7. If $G$ is a simple graph such that $V(G)$ can be partitioned into an (induced) independent set $J_{0}$ together with $s$ cliques $J_{1}, \ldots, J_{s}$, then $\operatorname{sreg}(S / I(G)) \leq s$.

Corollary 5.8. If $G$ is a simple graph such that $A \subset V(G)$ induces a clique, then

$$
\operatorname{sreg}(S / I(G)) \leq \operatorname{sreg}(S / I(G \backslash A))+1 .
$$

Corollary 5.9. If $G$ is a simple graph and $\beta(G)$ is the minimax matching number of $G$, then $\operatorname{sreg}(S / I(G)) \leq \beta(G)$.

The proofs for the above three corollaries are similar to those for [27, Theorems 2, 3 and 11], and we will not repeat them here.

Remark 5.10. It is not difficult to see that the invariant $\beta(G)$ in Corollary 5.9 is bounded above by $\operatorname{cosize}(I(\mathcal{C}))$; thus, the result we established in Theorem 5.6 is better in this situation.

Here are some additional applications of Theorem 5.6. Recall that an interval graph is an intersection graph of a family of intervals (closed, open or half-open) on the real line. Interval graphs are chordal. A cointerval graph is the complement of an interval graph. The boxicity of a graph $G$, denoted box $(G)$, is the cardinality of the smallest edge covering of $\bar{G}$ by co-interval spanning subgraphs (by an equivalent definition by [5, Corollary 3.1]). Thus, $\operatorname{cochord}(G) \leq \operatorname{box}(\bar{G})$. As a corollary to Theorem 5.6, we have

Corollary 5.11. If $G$ is a simple graph, then $\operatorname{sreg}(R / I(G)) \leq \operatorname{box}(\bar{G})$. 
Corollary 5.12. If $G$ is a co-planar graph, namely, if $\bar{G}$ is planar, then $\operatorname{sreg}(S / I(G)) \leq 3$.

Proof. It follows directly from the fact that $\operatorname{box}(\bar{G}) \leq 3([\mathbf{2 6}])$.

Remark 5.13. We are not sure whether the upper bound of the above inequality can be achieved as in [27, Proposition 18]. Notice that, for the special case when the graph $G=3 K_{2}$, it is clear that $\bar{G}$ is the 1skeleton of the Octahedron, hence planar. However, $\operatorname{sreg}(S / I(G))=2$ by Corollary 5.3 .

Remark 5.14. When $G$ is a simple graph, an induced matching in $G$ is a matching which forms an induced subgraph of $G$ and that indmatch $(G)$ denotes the number of edges in a largest induced matching. We have $\operatorname{reg}(S / I(G)) \geq \operatorname{indmatch}(G)$ by [17, Lemma 2.2]. Unfortunately, we do not have $\operatorname{sreg}(S / I(G)) \geq \operatorname{indmatch}(G)$. For instance, $\operatorname{sreg}\left(S / I\left(2 K_{2}\right)\right)=1<\operatorname{indmatch}\left(2 K_{2}\right)=2$.

5.2. Clutters. In this subsection, we will consider an upper bound of Stanley regularity in terms of combinatorial data from general clutters. To be more specific, let $\mathcal{C}$ be a clutter. Then a 2-collage for $\mathcal{C}$, as defined in [9], is a subset $C$ of edges with the property that, for each $e \in E(\mathcal{C})$, we can delete a vertex $v$ so that $e \backslash\{v\}$ is contained in some edge of $C$. In particular, when $\mathcal{C}$ is a uniform clutter, the condition for $C$ to be a 2-collage is equivalent to saying that, for any edge $e$ not in $C$, there is an edge $f \in C$ such that the cardinality of the symmetric difference of $e$ and $f$ is 2 .

Lemma 5.15. If $\left\{e_{1}\right\}$ is a 2 -collage for the clutter $\mathcal{C}$, then $\operatorname{sreg}(S / I(\mathcal{C}))$ $\leq\left|e_{1}\right|-1$.

Proof. It suffices to show that $\operatorname{sdepth}_{S}\left(I^{\vee}\right) \geq n-\left|e_{1}\right|+1$ where $I=I(\mathcal{C})$. Suppose the edge set is $E(\mathcal{C})=\left\{e_{i}: 1 \leq i \leq s\right\}$. For each edge $e_{i}$ of $\mathcal{C}$, there is a monomial prime ideal $P_{i}=\left\langle x_{j}: x_{j} \in e_{i}\right\rangle \subset S$. Now $I^{\vee}=\bigcap_{i=1}^{s} P_{i}$. Without loss of generality, we may assume that $\sum_{i=1}^{s} P_{i}=\left\langle x_{1}, \ldots, x_{n}\right\rangle$ and $P_{1}=\left\langle x_{1}, \ldots, x_{r}\right\rangle$ with $1 \leq r \leq n-1$. 
We will use $Y=\left\{x_{1}, \ldots, x_{r}\right\}$ as the splitting set in Construction 4.2 and define the rings $S^{\prime}$ and $S^{\prime \prime}$ accordingly. Now, $I^{\vee}=\bigoplus_{\tau \subset[s]} I_{\tau}$ is a decomposition of $I^{\vee}$ as a direct sum of $\mathbb{Z}^{n}$-graded $\mathbb{K}$-subspaces of $I^{\vee}$.

When $\tau=\emptyset, \operatorname{sdepth}_{S}\left(I_{\emptyset}\right)=\operatorname{sdepth}_{S^{\prime}}\left(I^{\vee} \cap S^{\prime}\right)+n-r \geq 1+n-r$ by Lemma 2.2 .

When $\tau \neq \emptyset$, we can write $I_{\tau}$ as $I_{\tau}=H_{\tau} \otimes_{\mathbb{K}} L_{\tau}$ where

$$
H_{\tau}=\frac{\bigcap_{j \notin \tau} P_{j}^{\prime}+\sum_{j \in \tau} P_{j}^{\prime}}{\sum_{j \in \tau} P_{j}^{\prime}}
$$

and $L_{\tau}=\bigcap_{j \in \tau} P_{j}^{\prime \prime}$. Since $e_{1}$ is a 2-collage for $\mathcal{C}, P_{1}^{\prime \prime}=0$ and each $P_{i}^{\prime \prime}$ is principal for $2 \leq i \leq s$. In particular, if $L_{\tau}$ is nonzero, then it is principal and $\operatorname{sdepth}_{S^{\prime \prime}} L_{\tau}=\operatorname{dim}\left(S^{\prime \prime}\right)=n-r$. Meanwhile, as in the proof of Theorem 4.1, we know $\operatorname{sdepth}_{S^{\prime}}\left(H_{\tau}\right) \geq 1$. Consequently, if $I_{\tau} \neq 0$, then $\operatorname{sdepth}_{S}\left(I_{\tau}\right) \geq \operatorname{sdepth}_{S^{\prime}}\left(H_{\tau}\right)+\operatorname{sdepth}_{S^{\prime \prime}}\left(L_{\tau}\right) \geq 1+n-r$ by [21, Lemma 1.2].

Now $\operatorname{sdepth}_{S}\left(I^{\vee}\right) \geq \min \left\{\operatorname{sdepth}_{S}\left(I_{\tau}\right): \tau \subsetneq[s]\right.$ and $\left.I_{\tau} \neq 0\right\} \geq 1+$ $n-r=1+n-\left|e_{1}\right|$.

Theorem 5.16. If $\left\{e_{1}, \ldots, e_{s}\right\}$ is a 2 -collage in the clutter $\mathcal{C}$, then

$$
\operatorname{sreg}(S / I(\mathcal{C})) \leq \sum_{i=1}^{s}\left(\left|e_{i}\right|-1\right) .
$$

Proof. As in the proof for [9, Theorem 1.2], for each edge $e_{i}$ in the assumption, we set $H_{i}$ to be the clutter consisting of all edges $e$ with $e \backslash\{v\} \subset e_{i}$ for some vertex $v$. Now $E(\mathcal{C})=\bigcup_{i=1}^{s} E\left(H_{i}\right)$ and each $H_{i}$ satisfies the condition of Lemma 5.15. Now, we apply Lemma 5.2 (a).

Acknowledgments. We thank Hailong Dao and Jay Schweig for helpful explanations during the preparation of the current work. We would also like to thank the anonymous referee for a careful reading and many helpful comments.

\section{REFERENCES}

1. Winfried Bruns and Jürgen Herzog, Cohen-Macaulay rings, Cambr. Stud. Adv. Math. 39, Cambridge University Press, Cambridge, 1993. 
2. Winfried Bruns, Christian Krattenthaler and Jan Uliczka, Stanley decompositions and Hilbert depth in the Koszul complex, J. Commut. Alg. 2 (2010), $327-357$.

3. Mircea Cimpoeaş, Some remarks on the Stanley depth for multigraded modules, Matematiche (Catania) 63 (2009), 165-171.

4. , Several inequalities regarding Stanley depth, Rom. J. Math. Comp. Sci. 2 (2012), 28-40.

5. Margaret B. Cozzens and Fred S. Roberts, Computing the boxicity of a graph by covering its complement by cointerval graphs, Discr. Appl. Math. 6 (1983), 217228.

6. Hailong Dao and Jay Schweig, Bounding the projective dimension of a squarefree monomial ideal via domination in clutters, Proc. Amer. Math. Soc. 143 (2015), 555-565.

7. Gabriel Andrew Dirac, On rigid circuit graphs, Abh. Math. Sem. Univ. Hamburg 25 (1961), 71-76.

8. Huy Tài Hà, Regularity of squarefree monomial ideals, in Connections between algebra, combinatorics, and geometry, Susan M. Cooper and Sean SatherWagstaff, eds., Springer Proc. Math. Stat. 76, Springer, New York, 2014.

9. Huy Tài Hà and Russ Woodroofe, Results on the regularity of square-free monomial ideals, Adv. Appl. Math. 58 (2014), 21-36.

10. Jürgen Herzog, A generalization of the Taylor complex construction, Comm. Alg. 35 (2007), 1747-1756.

11. , A survey on Stanley depth, in Monomial ideals, computations and applications, Lect. Notes Math. 2083, Springer, New York, 2013.

12. Jürgen Herzog and Takayuki Hibi, Monomial ideals, Grad. Texts Math. 260, Springer-Verlag, London, 2011.

13. Jürgen Herzog, Dorin Popescu and Marius Vladoiu, Stanley depth and size of a monomial ideal, Proc. Amer. Math. Soc. 140 (2012), 493-504.

14. Jürgen Herzog, Marius Vladoiu and Xinxian Zheng, How to compute the Stanley depth of a monomial ideal, J. Algebra 322 (2009), 3151-3169.

15. Muhammad Ishaq and Muhammad Imran Qureshi, Upper and lower bounds for the Stanley depth of certain classes of monomial ideals and their residue class rings, Comm. Alg. 41 (2013), 1107-1116.

16. Gil Kalai and Roy Meshulam, Intersections of Leray complexes and regularity of monomial ideals, J. Combin. Theor. 113 (2006), 1586-1592.

17. Mordechai Katzman, Characteristic-independence of Betti numbers of graph ideals, J. Combin. Theor. 113 (2006), 435-454.

18. Kuei-Nuan Lin and Jason McCullough, Hypergraphs and regularity of square-free monomial ideals, Inter. J. Alg. Comput. 23 (2013), 1573-1590.

19. Gennady Lyubeznik, On the arithmetical rank of monomial ideals, J. Alg. 112 (1988), 86-89.

20. Marcel Morales, Ali Akbar Yazdan Pour and Rashid Zaare-Nahandi, The regularity of edge ideals of graphs, J. Pure Appl. Alg. 216 (2012), 2714-2719. 
21. Adrian Popescu, Special Stanley decompositions, Bull. Math. Soc. Sci. Math. Roum. 53 (2010), 363-372.

22. Tim Römer, Generalized Alexander duality and applications, Osaka J. Math. 38 (2001), 469-485.

23. Ali Soleyman Jahan, Prime filtrations and Stanley decompositions of squarefree modules and Alexander duality, Manuscr. Math. 130 (2009), 533-550.

24. Richard P. Stanley, Linear Diophantine equations and local cohomology, Invent. Math. 68 (1982), 175-193.

25. Naoki Terai, Alexander duality theorem and Stanley-Reisner rings, Surik. Kokyuroku 1078 (1999), 174-184.

26. Carsten Thomassen, Interval representations of planar graphs, J. Combin. Theor. 40 (1986), 9-20.

27. Russ Woodroofe, Matchings, coverings, and Castelnuovo-Mumford regularity, J. Comm. Alg. 6 (2014), 287-304.

28. Kohji Yanagawa, Alexander duality for Stanley-Reisner rings and squarefree $\mathbf{N}^{n}$-graded modules, J. Alg. 225 (2000), 630-645.

Wu Wen-Tsun Key Laboratory of Mathematics of CAS and School of Mathematical Sciences, University of Science and Technology of China, Hefei, Anhui, 230026, P.R. China

Email address: yhshen@ustc.edu.cn 